\title{
Processing Facial Expressions That Conflict With Their Meanings to an Observer: An Event Related Potential Study
}

\author{
Qiwei Yang, Yuping Zhang, Jianfeng Wang* and Yan $W u^{*}$ \\ Sichuan Research Center of Applied Psychology, Chengdu Medical College, Chengdu, China
}

As social signals, identical facial expressions can be perceived differently, even oppositely, depending on the circumstances. Fast and accurate understanding of the information conveyed by others' facial expressions is crucial for successful social interaction. In the current study, we used electroencephalographic analysis of several event-related potentials (ERPs) to investigate how the brain processes the facial expressions of others when they

OPEN ACCESS

Edited by:

Jonathan Levy,

Aalto University, Finland

Reviewed by:

Antonios I. Christou, University of Cyprus, Cyprus

Chao Liu,

Beijing Normal University, China

${ }^{*}$ Correspondence:

Jianfeng Wang

wifzy1985@163.com

Yan Wu

wuyanzhicheng@163.com

Specialty section: This article was submitted to

Emotion Science,

a section of the journal

Frontiers in Psychology

Received: 07 February 2020

Accepted: 14 May 2020

Published: 17 June 2020

Citation:

Yang $Q$, Zhang $Y$, Wang $J$ and Wu $Y$ (2020) Processing Facial Expressions

That Conflict With Their Meanings to

an Observer: An Event Related

Potential Study.

Front. Psychol. 11:1273.

doi: 10.3389/fpsyg.2020.01273 indicate different self-outcomes. In half of the trial blocks, a happy face indicated "Win" and an angry face indicated "Lose." In the other half of the blocks, the rule was reversed. The results showed that the N170 could distinguish expression valence and the N300 could distinguish outcome valence. The valence of the expression (happy or angry) and the valence of the outcome (Win or Loss) interacted with each other in the early, automatic perceptual processing stage (N1) as well as in the later, cognitive evaluation stage (P300). Standardized Low-Resolution Electromagnetic Tomography (SLORETA) results indicated that the N1 modulation only occurred for happy faces, which may relate to automatic emotion regulation, while the interaction on P300 was significant only for angry faces, which might be associated with the regulation of negative emotions.

Keywords: facial expression, outcome evaluation, event-related potential, N1, P3

\section{INTRODUCTION}

The facial expressions of others convey information that is important for social communication. The processing of facial expression has been found to be strongly modulated by situational context such as the emotional valence of background images (Carroll and Russell, 1996), the meaning conveyed by stories accompanying facial expressions (Righart and de Gelder, 2006), and the race (Herzmann et al., 2013), attractiveness (Liang et al., 2010), and trustworthiness (Ruz et al., 2013) of people whose faces are being viewed.

As a social signal, the same facial expression can be perceived differently depending on these influencing factors. This phenomenon could be assumed in two ways. First, how much does the expression on a perceived face influence the attentional resources that it can attract? For example, when someone is in a singing competition, even though the audience includes hundreds of faces, the judges' faces are the center of one's attention because their facial expressions are valid predictors of one's score. Second, what is the relationship between the 
valence of the expression itself and the valence of the meaning it conveys? A happy face of a partner indicates one's team is winning. In this case, both the valence of the facial expression and the valence of its outcome to him/her are positive. However, when the face of one's opponent is frustrated, its outcome is also positive for him/her, despite the negative valence of the expression itself. In order to integrate a facial expression in a particular outcome, we must check whether its outcome valence and its specific emotional valence are contextually appropriate. According to previous studies, the processing and decoding of facial expressions of emotion involves a double check of valence and specific emotional information for the perceiver (Aguado et al., 2013, 2019). However, how the valence of a perceived emotion and the valence of the self-outcome it conveys are processed in the brain has not yet been explored.

The main goal of the present study is to investigate how the brain processes the facial expressions of others when they indicate different self-outcomes with electrophysiological recording. In the current study, different valences of facial expressions (happy and angry) were used to indicate the outcomes in a monetary gambling game. A participant was presented with two rectangles on the screen, one associated with a positive outcome (Win) and the other associated with a negative outcome (Loss). After they selected a rectangle, a picture of facial expression would appear to reveal the outcome. In half of the trial blocks, a happy face indicated "Win" and an angry face indicated "Lose." In the other half of the blocks, the rule was reversed. Four conditions were created: (matched conditions) valence of the face and valence of the outcome were both positive or both negative (Happy face indicated Win; Angry face indicated Loss); (mismatched conditions) valences were opposite (Happy face indicated Loss; Angry face indicated Win). Before each block, participants were instructed as to which pairing would be used. The even-related potentials (ERPs) obtained during these different conditions were then compared.

Based on the abundant evidence from affective priming studies (Fazio et al., 1986; Moors and De Houwer, 2001; Klauer and Musch, 2003), we assume that the valence of perceived emotion checking is automatic, taking place at early processing stages. According to previous studies about outcome evaluation (Wu and Zhou, 2009; Yang et al., 2018), we assume that the valence of outcome checking is intentional, taking place at later processing stages. A general prediction that directly follows this account is that the valence matching between facial expressions (happy/angry) and outcome (win/lose) should have differential effects on the processing of positive and negative expressions.

Numerous ERP studies have investigated the time course of facial expression processing (Werheid et al., 2005; Trautmann et al., 2009; Vlamings et al., 2009; Lassalle and Itier, 2013; Zhang et al., 2013; Recio et al., 2014; Yuan et al., 2014). Several ERP components have been consistently observed. N100 (the fronto-central distributed negative component) and P100 (the parietal positive component) reflect very fast, automatic early perceptual processing of faces. N170 (the negative parietal-occipital component) is specifically elicited by faces and is sensitive to affective valence. The fronto-central vertex-positive potential (VPP), N300, and P300 are components that reflect the differentiation and evaluation of various facial expressions (Luo et al., 2010). The present study hypothesized that among the ERP components usually elicited by facial expressions, P300 would be selectively modulated by the outcome (Win or Lose). P300 is often modulated by the emotional or arousing content of stimuli. Studies have shown that compared with neutral stimuli, emotional stimuli enhanced the P300 component, and this modulation was stronger for highly arousing stimuli (Carretie et al., 1997; Cramer, 1998). Additionally, this component is thought to reflect evaluative processing, such that its amplitude increases when more cognitive resources are allocated (Friedman et al., 2001; Wu and Zhou, 2009; Asaumi et al., 2014; Roca et al., 2015). We assume that in the conditions for which the two valences are inconsistent, increased cognitive resources would be demanded, which would contribute to a larger P300 than when the two valences were consistent. We also hypothesize that ERP discrimination of the expressions would be earlier than that of the outcomes because the participants need to recognize the expression before they can know the outcome. Further, a recent ERP study found that early perceptual components such as $\mathrm{P} 100$ were also sensitive to social-emotional regulation, supporting the flexibility and modifiability of early ERP components (Beckes et al., 2013). Therefore, we hypothesize that an interaction between the two valences also occur between the early components such as $\mathrm{N} 1$ and $\mathrm{P} 1$.

\section{MATERIALS AND METHODS}

\section{Participants}

Twenty right-handed participants with no history of neurological disorders, brain injury, or developmental disabilities participated in the experiment. All had normal or corrected-to-normal vision. The study was approved by the Medical Ethical Committee of Shenzhen University. All participants provided their written informed consent. Data from two participants were excluded because the percentage of bad electroencephalographic (EEG) epochs was too high (35\%). Thus, 18 participants were included in the final analysis (10 men; age: $24.95 \pm 0.65$ years).

\section{Stimuli}

The stimuli used comprised 120 photos of faces from the native Chinese Facial Affective Picture System (CFAPS), including 60 happy faces and 60 angry faces. The recognition consistency was $86.64 \pm 8.38 \%$ for happy expressions and $83.77 \pm 6.56 \%$ for angry expressions. The intensity of happy and angry expressions was $6.43 \pm 0.86$ and $6.78 \pm 0.69$, respectively. No significant differences of recognition accuracy or intensity were found between the two categories of faces $(p>0.5)$. Faces of men and women were represented equally. Happy and angry faces were identical to each other in size, background, contrast grade, brightness, and other physical properties. All faces were gray-scale and were presented on a black background $\left(3.0^{\circ} \times 3.5^{\circ}\right.$ visual angle). 


\section{Experimental Procedures}

Stimulus presentation and behavioral data acquisition were performed using E-Prime software (Version 1.0, Psychology Software Tools, Inc.). During the task, participants sat comfortably in an electrically-shielded room approximately $100 \mathrm{~cm}$ from a 15-inch color computer monitor. Each trial began with the individual presentation of two gray rectangles $\left(2.3^{\circ} \times 3.2^{\circ}\right.$ of visual angle), which indicated two alternative options on the left and right sides of a fixation point. The participant was informed that one rectangle corresponded to a "Win" and the other to a "Loss." The participant was asked to gamble by pressing the " $F$ " or "J" key on a keyboard with their index fingers to choose one rectangle. The rectangles remained on the screen until the participant chose a side. Next, a blank interval lasting 400-700 ms (randomly) was presented, followed by the presentation of a face at the chosen location that represented the outcome. The photo remained on the monitor for $800 \mathrm{~ms}$. The inter-trial interval varied from 1,500 to $2,500 \mathrm{~ms}$ (see Figure 1).

Trials were presented in four blocks of 120 trials (total 480 trials). Before two of the four blocks, participants were informed that a happy face indicated a "Win" and an angry face indicated a "Loss." In the other two blocks, they were told the reverse. Block order was counterbalanced across participants. Participants were informed that each trial was worth 10 renminbi (RMB) (i.e., they could win or lose $10 \mathrm{RMB}$ on each trial).

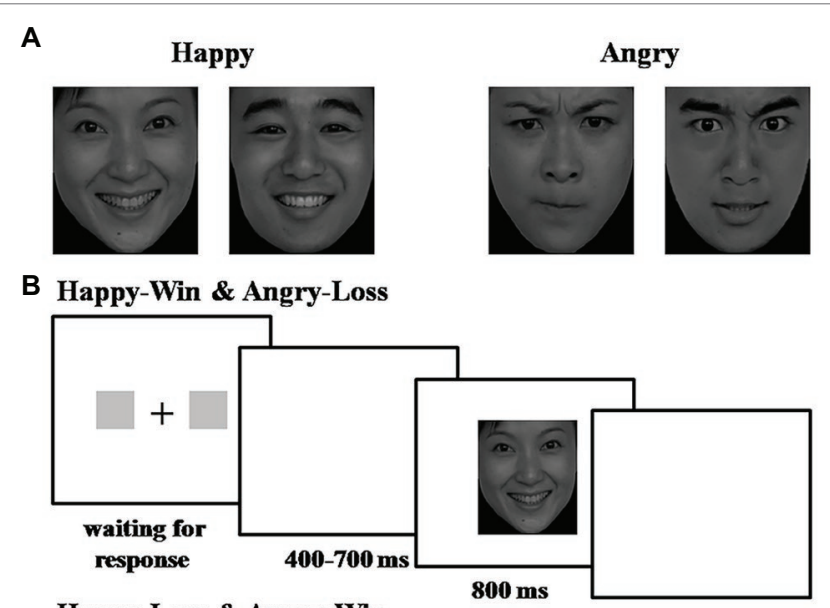

C Happy-Loss \& Angry-Win

$1500-2500 \mathrm{~ms}$

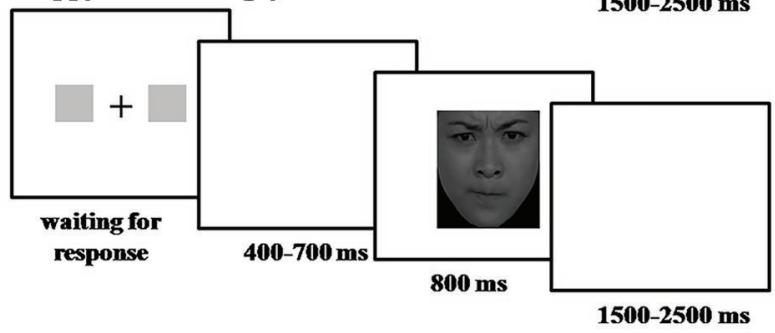

FIGURE 1 | Experimental procedure and stimuli. (A) Examples of the photos used: (left) happy male/female faces; (right) angry male/female faces. The faces were selected from the revised version of the Chinese Facial Affective Picture System (CFAPS). (B) Experimental procedure for Happy-Win and Angry-Loss blocks. (C) Experimental procedure for Happy-Loss and Angry-Win blocks.
We used a $2 \times 2$ within subject experimental design. The first factor was the valence of the facial expressions: Happy or Angry. The second factor was the valence of the outcome indicated by the face: Win or Loss. There were four conditions: Happy-Win, Happy-Loss, Angry-Win, and Angry-Loss.

Before the experiment, the task, its rules, and meaning of the faces were explained to the participants. Additionally, they were told that the higher the points they earned, the more bonus money they would receive at the end of the experiment. However, after the task, they were briefed that their total gains and losses were balanced.

\section{Electroencephalography Acquisition and Analysis}

Electroencephalographic data were recorded from a 64-electrode scalp cap using the 10-20 system (Brain Products, Munich, Germany) with the reference on the left and right mastoids. A vertical electrooculogram (EOG) was recorded with electrodes placed above and below the left eye. EEG and EOG data were amplified, band-pass filtered (0.01-100 Hz), and sampled at $500 \mathrm{~Hz}$. All electrode impedances were maintained below $5 \mathrm{k} \Omega$.

EEG data were pre-processed and analyzed using MATLAB R2011b (Math Works, US) and EEGLAB toolbox (Delorme and Makeig, 2004). EEG data at each electrode were downsampled to $250 \mathrm{~Hz}$, re-referenced to the grand average, and band-pass filtered (0.01-30 Hz). EEG data from $200 \mathrm{~ms}$ before until $800 \mathrm{~ms}$ after the onset of the facial stimuli were extracted. In order to discard data that was contaminated by EOG artifacts, the data were decomposed by extended infomax ICA using binica, as implemented in EEGLAB (Jung et al., 2001). Epochs with amplitude values exceeding $\pm 50 \mu \mathrm{V}$ at any electrode were excluded from the average.

\section{Data Measurement and Analysis}

We mainly analyzed the ERP elicited by happy and angry faces. The averaged epoch was $1,000 \mathrm{~ms}$, including a $200 \mathrm{~ms}$ pre-stimulus baseline. In this study, the amplitudes of N1, P1, VPP, N170, N300, and P300 components were measured and analyzed. Based on the topographical distribution of the grand-averaged ERP activity and previous studies (Righart and de Gelder, 2006; Williams et al., 2006; Luo et al., 2010), different sets of electrodes for each component were chosen. Fz, F3, F4, FCz, FC3, and FC4 electrode sites were selected for the analysis of $\mathrm{N} 1$ (90-140 ms) and VPP (140-220 ms); Pz, P3, P4, POz, PO3, and $\mathrm{PO} 4$ were selected for the analysis of $\mathrm{P} 1$ component (100-160 ms); N170 component (140-200 ms) was analyzed at the P7, P8, PO7, and PO8 electrode sites; N300 component (250-400 ms) was analyzed at the T7, T8, FT7, and FT8 electrode sites; and 10 electrode sites $(\mathrm{Cz}, \mathrm{C} 3, \mathrm{C} 4, \mathrm{CPz}, \mathrm{CP} 3$, $\mathrm{CP} 4, \mathrm{Pz}, \mathrm{P} 3, \mathrm{P} 4$, and $\mathrm{POz}$ ) were selected for the statistical analysis of P300 component (300-500 ms). A three-way repeated measure analysis of variance (ANOVA) on the amplitude of each component was conducted with Face pictures (two levels: Happy, Angry), Outcome (two levels: Win, Loss), and Electrode site as within-subject factors. Degrees of freedom for F-ratios were corrected according to the Greenhouse-Geisser method. 
Statistical differences were considered significant at $p<0.05$; posthoc comparisons were Bonferroni-corrected at $p<0.05$.

\section{sLORETA Analysis}

We used Standardized Low-Resolution Electromagnetic Tomography (sLORETA) to determine the sources of the differences that we found in the N100 and P300 components. sLORETA is a functional imaging method based on certain EEG and neuroanatomical constraints (Pascual-Marqui et al., 1994). It computes images of electrical activity from the EEG data in a realistic head model using the MNI152 template and estimates the three-dimensional distribution of the current density within 6,239 voxels at a spatial resolution of $5 \mathrm{~mm}$. This method has been established as useful for determining deep structures such as the ACC and others within the temporal lobe (Pizzagalli et al., 2004; Zumsteg et al., 2006).

For the current dataset, in order to localize the brain structures responsible for the effects we observed on N100 and $\mathrm{P} 300$, a $t$-test was performed for the current densities on different conditions for $\mathrm{N} 100$ and $\mathrm{P} 300$ in their respective time windows (N100: 90-140 ms; P300: 300-500 ms), employing a LOT-F-ratio statistics for paired groups (HappyLoss > Happy-Win for N100 and Angry-Win > Angry-Loss for P300, separately), with 5,000 bootstrapping and a level of significance of $p<0.05$.

\section{RESULTS}

\section{N100}

The Face (Happy vs. Angry) $\times$ Outcome (Win vs. Loss) interaction was significant for N100 amplitude $\left[F_{(1,17)}=5.433\right.$, $\eta_{\mathrm{p}}{ }^{2}=0.242$, and $\left.p=0.032\right]$. The pairwise comparisons revealed that when the facial expression was Happy, Losses elicited significantly greater negative amplitude than Wins $(-2.781 \mu \mathrm{V}$ for Happy-Loss and $-2.336 \mu \mathrm{V}$ for Happy-Win, $p=0.005$ ). The difference between Win and Loss was not significant for angry faces $(-2.518 \mu \mathrm{V}$ for Angry-Loss and $-2.525 \mu \mathrm{V}$ for Angry-Win, $p=0.0968$; see Figure 2).

\section{N170}

We found a significant main effect of face on N170 amplitude such that angry faces elicited significant larger amplitudes than happy faces. [Happy: $-5.922 \mu \mathrm{V}$; Angry: $-6.258 \mu \mathrm{V}$; $F_{(1,17)}=7.457, \eta_{\mathrm{p}}{ }^{2}=0.305$, and $\left.p=0.014\right]$. We did not find a main effect of outcome or an interaction between Face and Outcome (see Figure 3A).

\section{N300}

We found a significant main effect of Outcome for N300 amplitude such that Wins elicited significantly greater negative amplitudes than Losses [Wins: $-4.177 \mu \mathrm{V}$; Losses: $-3.710 \mu \mathrm{V}$; $F_{(1,17)}=10.848, \eta_{\mathrm{p}}{ }^{2}=0.390$, and $p=0.004$; see Figure $\left.3 \mathrm{~B}\right]$.

\section{P300}

We found a significant main effect of Outcome on P300 amplitude. Wins elicited significant larger amplitudes than Losses [Wins: $4.257 \mu \mathrm{V}$; Losses: $3.950 \mu \mathrm{V} ; F_{(1,17)}=11.004, \eta_{\mathrm{p}}{ }^{2}=0.393$, and $p=0.004]$. We also found a significant main effect of Electrode $\left[F_{(9,153)}=6.618, p<0.001\right]$. Specifically, FCz, FC3, FC4, Cz, C3, $\mathrm{C} 4$, and $\mathrm{Pz}$ electrodes elicited larger amplitudes than the others $(p<0.05)$. Additionally, we found that the three-way interaction of Face $\times$ Outcome $\times$ Channel was significant $\left[F_{(9,153)}=3.283\right.$, $\eta_{\mathrm{p}}{ }^{2}=0.162$, and $\left.p=0.016\right]$. Pairwise comparison revealed that significantly larger amplitudes occurred for angry faces on Wins

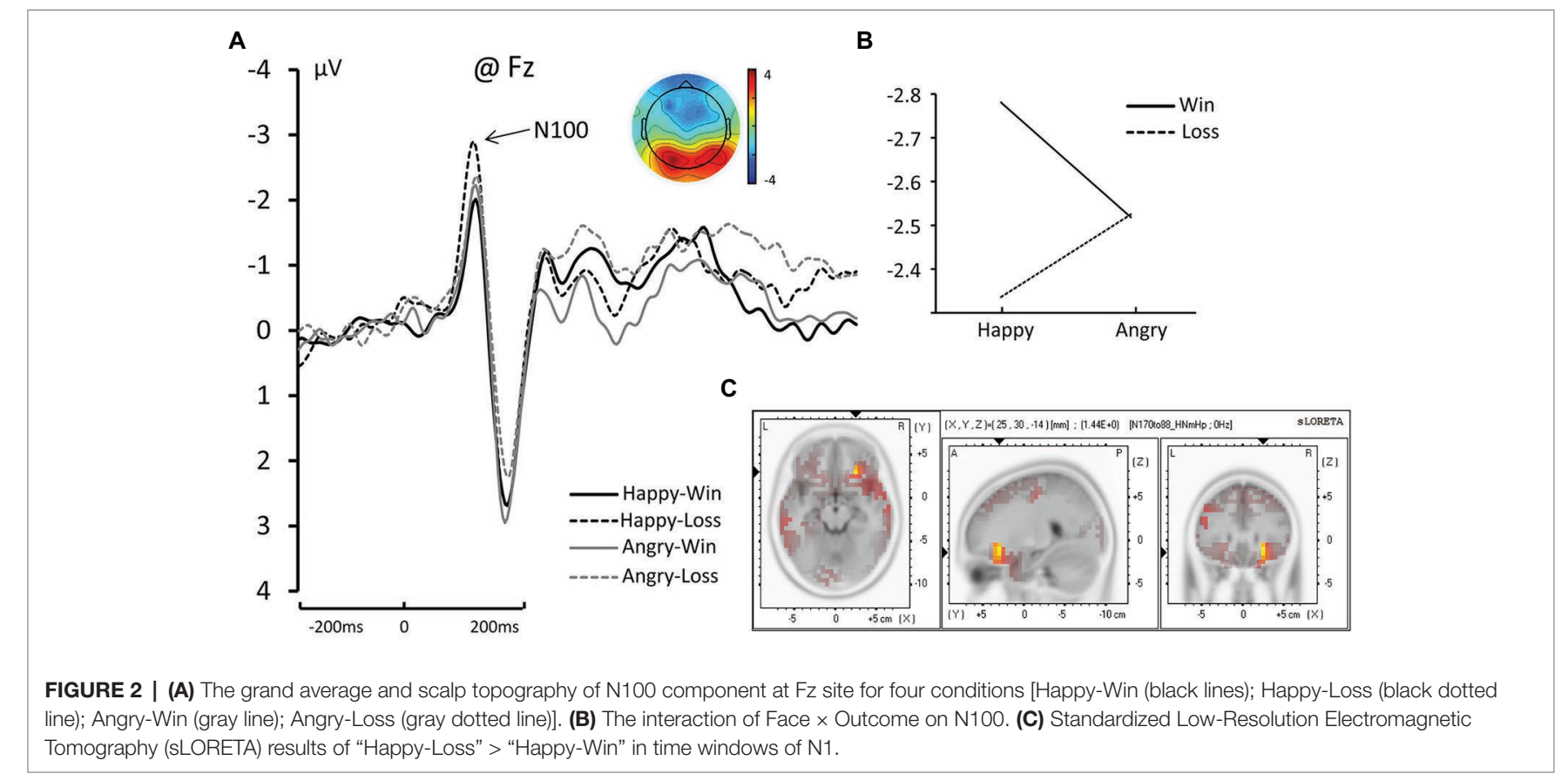




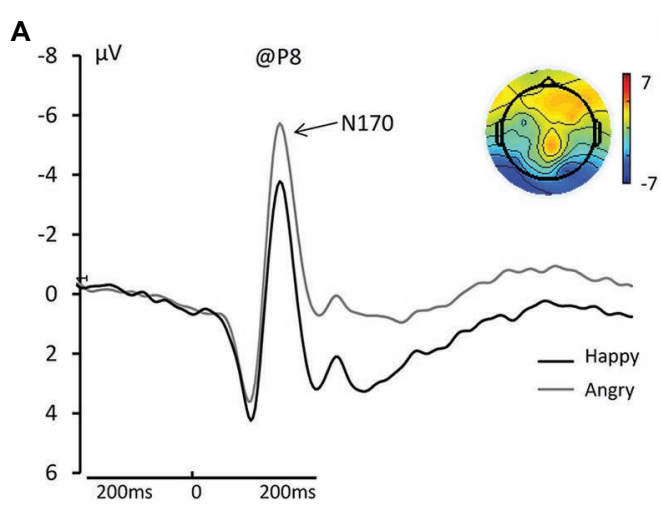

B

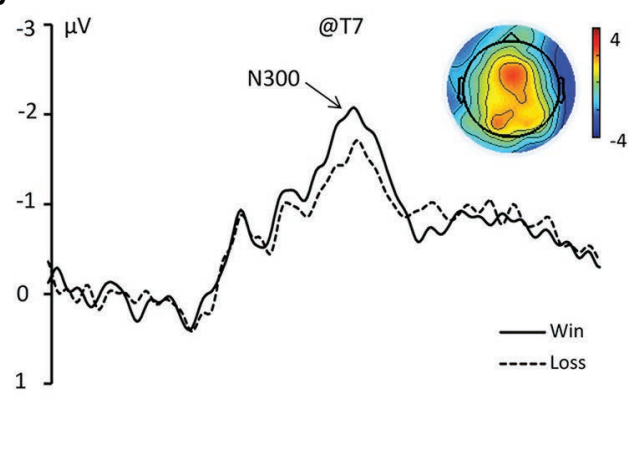

FIGURE 3 | (A) The grand average and scalp topography of N170 component at P8 site for two conditions [Happy (black lines); Angry (gray line)]. (B) The grand average and scalp topography of N300 component at T7 site for two conditions [Win (black lines); Loss (black dotted line)].

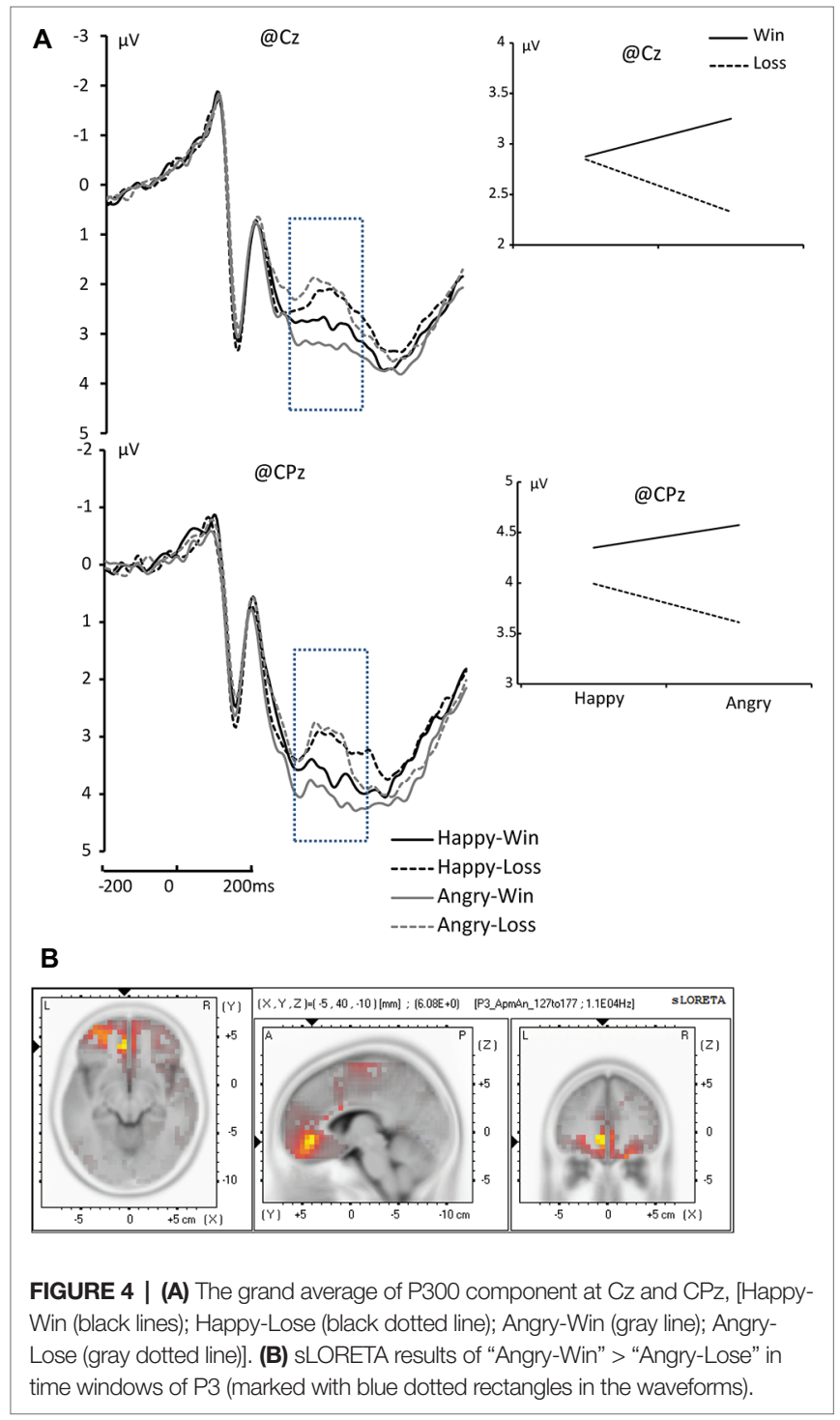

than on Losses at Cz, C3, C4, CPz, and CP3 ( $p=0.001, p=0.006$, $p=0.031, p=0.013$, and $p=0.004$, respectively; see Figure 4A).
We did not find any significant main effects or interactions for other ERP components.

\section{Standardized Low-Resolution Electromagnetic Tomography}

The analyses revealed a difference in the inferior frontal gyrus and middle frontal gyrus (BA47/BA11) between Happy-Loss and Happy-Win conditions within the N1 time window such that the Happy-Loss condition resulted in significantly higher current density than the Happy-Win condition [Montreal Neurological Institute $(\mathrm{MNI})$ coordinates $=(25,30,-14), p=0.017$; see Figure 2C]. The Angry-Win resulted in significantly higher activation in the anterior cingulate cortex (BA32) and orbitofrontal region (BA11) than did the Angry-Lose condition within the P300 time window (MNI $=-5,40,-10, p=0.018$; Figure 4B).

\section{DISCUSSION}

This study explored how the brain processes facial expressions that indicate monetary outcome for oneself. Our results show that the brain first distinguishes the valence of the expression (happy from angry in our case), as reflected in a significant main effect of the facial expression in the N170 component. The brain also distinguishes the outcome, as reflected in the observed significant main effect of outcome on N300. The processing of self-outcome interacted with the processing of facial expression in the early automatic stage and also in the later evaluative stage, as reflected by the observed significant interactions that affected N1 and P300 amplitude.

\section{The Interaction in the Early Automatic Stage of Processing: N100}

In the frontal $\mathrm{N} 1$, we found a significant interaction of Face $\times$ Outcome in which the difference in amplitude between Win and Loss was significant for a happy face, but not for an angry face. Specifically, the Happy-Loss condition elicited a significantly larger negativity than the Happy-Win condition. This result indicates that the outcome for the observer began 
to interact with the perception of the facial expression at a very early, automatic stage. The enhancement during the HappyLose condition could be related to an initial amplification of relevant processing generated by top-down factors (Hillyard and Picton, 1987; Ruz et al., 2013). In the mismatched blocks, facial expressions were not tied to their natural meanings. Therefore, the recognition of the outcome from the facial expression was likely associated with higher processing demands, which requires the allocation of more cognitive resources (Williams et al., 2003). The sLORETA results revealed a significant difference between Happy-Loss and Happy-Win which was related to higher activation in the orbitofrontal cortex (BA11) in the Happy-Lose condition than in the Happy-Win condition. This brain region has been found to be specifically activated in automatic emotion regulation (ER) (Ochsner and Gross, 2005; Phillips et al., 2008; Etkin et al., 2011; Hallam et al., 2015). The automatic ER seems to underlie this processing. ER refers to the processes involved in the initiation, maintenance, and modification of the occurrence, intensity, and duration of feeling states (Gross and Levenson, 1993; Eisenberg et al., 2000). Automatic ER specifically means the ER with features of automaticity (i.e., immediacy, efficiency, and redundancy of conscious intent (Gollwitzer and Sheeran, 2006). The brain regions that support automatic ER include medial frontal areas such as the medial orbitofrontal cortex (mOFC). Based on the literature, the mOFC is a heteromodal association area that unites information from the sensory modalities, representations of past experiences, and the processing of contextually-relevant information (Hallam et al., 2015). It is thus suitable for handling the expressions of others that convey information with changing valences, which requires the integration of multiple types of information, such as those from sensory input, experience, and social contexts.

Why the Win/Loss difference was only significant for happy faces was not immediately clear. It might be related to the lower priority that happy faces have in social interactions. Studies suggest that angry expressions are initially prioritized by our cognitive system because we benefit from early detection of potential threats in the environment (Fox et al., 2000; Avero and Calvo, 2006). However, unlike detection tasks, happy expressions show clear advantages in recognition tasks. Happy faces were found to be recognized faster and more accurately (Leppanen and Hietanen, 2004). The same study also found that a smiling mouth became visually salient very early ( $95 \mathrm{~ms})$, which corresponds temporally with the N100 (Calvo et al., 2014). Another study showed that among all expressions, only recognition of happy expressions was unaffected by the intensity of the expressions-even low intensity happy faces were recognized with nearly $100 \%$ accuracy (Hess et al., 1997). In situations in which happy faces indicate a negative outcome, they would likely be quickly recognized and then modulated through automatic ER. Angry faces might not yet be recognized during this time window.

\section{The Discrimination of the Two Valences: N170 and N300}

$\mathrm{N} 170$ is a negative-going component detected at the occipitotemporal electrode sites that peaks around $170 \mathrm{~ms}$ post-stimulus. The component clearly distinguishes faces from non-face visual stimuli. However, evidence regarding whether N170 is responsive to emotional expression is conflicted; while some studies found that N170 did not discriminate emotional expressions (Luo et al., 2010; Nakajima et al., 2012), others found that it did (Batty and Taylor, 2003; Miyoshi et al., 2004; Lynn and Salisbury, 2008; Herbert et al., 2013). In particular, N170 amplitude has been reported to differ between happy and angry faces (Krombholz et al., 2007). In line with these latter studies, here we found a main effect of facial expression on N170 in which angry faces elicited significantly larger amplitudes than happy faces.

After decoding the facial expressions, the valence of the outcome could be distinguished via the N300 component; we found significantly larger negative amplitude for Wins than for Losses. The N300 largely reflects the dimensionality of the affective valence in higher-level phases of cognitive processing, such as stimulus evaluation and selection (Carretie et al., 2001a,b; Campanella et al., 2002; Luo et al., 2010). In the current study, the participants needed to mentally recognize and label the presented facial expressions, then deduce the monetary outcome. Thus, a main effect of facial expression (N170) before a main effect of outcome (N170) was a reasonable observation.

\section{The Interaction in the Evaluation Stage: P300}

Scientists believe that P300 is involved in a large number of cognitive and affective processes and it is traditionally associated with the allocation of mental resources (Olofsson et al., 2008). When a facial expression contains information that is important to an observer (e.g., monetary gain or loss), it usually draws more attention and requires more cognitive resources to analyze and evaluate. Interestingly, in the current study, we found that during the P300 time window, the positive and negative facial expressions were evaluated differently under different conditions. A three-way interaction of Face $\times$ Outcome $\times$ Channel was observed. The difference between Angry-Win and Angry-Loss was significant in the central regions. sLORETA results found that regions that were differentially activated between Angry-Win and Angry-Loss were localized in the ACC (BA32) and orbitofrontal region (BA11). These regions have been found to be responsible for the regulation of negative emotions (Levesque et al., 2003; Ochsner et al., 2004, 2012; Phan et al., 2005; Mak et al., 2009). In the current design, in the blocks where the angry face indicated a positive outcome, the participant may need to suppress the negative affect aroused by the naturally negative stimulus and re-identify the face as positive. Thus, recruiting neural circuits related to the regulation of negative affect is unsurprising for this condition.

Other studies have shown that valence can also affect later components, such as, P3 (Olofsson et al., 2008). Interestingly, we did not observe a significant effect of facial expression on the ERPs for which a main effect of expression has often been found (e.g., N300 and P300). We assume this was because the most important information for the participants was not the expressions themselves, but the monetary outcome. Therefore, after recognizing the expressions in the N200 time window, processing of the outcome likely 
dominated and the effect of the expressions during the N300 and P300 time windows would be weakened.

Actually, ERP studies have produced ambiguous results on the time course of face and valence processing. Some research have found that the $\mathrm{P} 1$ and $\mathrm{N} 1$ can be modulated by emotional valence (Levesque et al., 2003; Ochsner et al., 2004, 2012; Phan et al., 2005; Mak et al., 2009). Rellecke and colleagues found that automatic enhanced encoding of angry faces were indicated by P1, N170, and EPN in the early processing stages. However, our results only found the main effect of emotional valence in N170 and the valence and outcome interactions with the processing of other's facial expression in an early automatic stage (Levesque et al., 2003; Ochsner et al., 2004, 2012; Phan et al., 2005; Mak et al., 2009). Let us note that this early P1 modulation by emotion is debated as many studies also failed to report modulations of the P1 by facial expressions of emotion (Levesque et al., 2003; Ochsner et al., 2004, 2012; Phan et al., 2005; Mak et al., 2009). We assumed that the reason is that these components are related to differentiation of certain expressions (Olofsson et al., 2008), which should occur after valence processing according to the dimensional model.

In conclusion, the current investigation explored how facial expression stimuli are processed when they indicate positive or negative outcomes for those observing them. The results suggest that early perceptual processing of facial expression is influenced by the valence of outcomes, as evidenced by an enhanced N100 component when happy faces indicate a financial loss. Subsequently, the valence of the face is decoded by the N170 component and the valence of the outcome is discriminated by the N300 component. At a later cognitive evaluation stage, the face and outcome valences interact again, as evidenced by the differences in the P300 component between financial gains and losses represented by angry faces. This interaction may reflect the regulation of emotional responses that are elicited by negative stimuli when the stimuli indicate positive outcomes.

\section{REFERENCES}

Aguado, L., Dieguez-Risco, T., Méndez-Bértolo, C., Pozo, M. A., and Hinojosa, J. A. (2013). Priming effects on the N400 in the affective priming paradigm with facial expressions of emotion. Cogn. Affect. Behav. Neurosci. 13, 284-296. doi: 10.3758/s13415-012-0137-3

Aguado, L., Dieguez-Risco, T., Villalba-Garcia, C., and Hinojosa, J. A. (2019). Double-checking emotions: valence and emotion category in contextual integration of facial expressions of emotion. Biol. Psychol. 146:107723. doi: 10.1016/j.biopsycho.2019.107723

Asaumi, Y., Morita, K., Nakashima, Y., Muraoka, A., and Uchimura, N. (2014). Evaluation of P300 components for emotion-loaded visual event-related potential in elderly subjects, including those with dementia. Psychiatry Clin. Neurosci. 68, 558-567. doi: 10.1111/pcn.12162

Avero, P., and Calvo, M. G. (2006). Affective priming with pictures of emotional scenes: the role of perceptual similarity and category relatedness. Span. J. Psychol. 9, 10-18. doi: 10.1017/S1138741600005928

Batty, M., and Taylor, M. J. (2003). Early processing of the six basic facial emotional expressions. Brain Res. Cogn. Brain Res. 17, 613-620. doi: 10.1016/ S0926-6410(03)00174-5

Beckes, L., Coan, J. A., and Morris, J. P. (2013). Implicit conditioning of faces via the social regulation of emotion: ERP evidence of early attentional biases for security conditioned faces. Psychophysiology 50, 734-742. doi: 10.1111/psyp.12056
The sample size $(n=18)$ was a limitation of the current study as it is relatively small for an ERP study. Our findings should therefore be validated using larger sample sizes.

\section{DATA AVAILABILITY STATEMENT}

The datasets generated for this study are available on request to the corresponding author.

\section{ETHICS STATEMENT}

The studies involving human participants were reviewed and approved by the Medical Ethical Committee of Shenzhen University. The patients/participants provided their written informed consent to participate in this study.

\section{AUTHOR CONTRIBUTIONS}

Conceived and designed the experiments: QY and YW. Performed the experiments: YZ and JW. Analyzed the data: JW. Wrote the manuscript: QY and YW.

\section{FUNDING}

This study was funded by the National Natural Science Foundation of China (31671173, 31701001, and 31700980).

\section{ACKNOWLEDGMENTS}

We thank Dr. X. R. Zhu for discussions.
Calvo, M. G., Beltran, D., and Fernandez-Martin, A. (2014). Processing of facial expressions in peripheral vision: neurophysiological evidence. Biol. Psychol. 100, 60-70. doi: 10.1016/j.biopsycho.2014.05.007

Campanella, S., Quinet, P., Bruyer, R., Crommelinck, M., and Guerit, J. M. (2002). Categorical perception of happiness and fear facial expressions: an ERP study. J. Cogn. Neurosci. 14, 210-227. doi: 10.1162/ 089892902317236858

Carretie, L., Iglesias, J., Garcia, T., and Ballesteros, M. (1997). N300, P300 and the emotional processing of visual stimuli. Electroencephalogr. Clin. Neurophysiol. 103, 298-303. doi: 10.1016/S0013-4694(96)96565-7

Carretie, L., Martin-Loeches, M., Hinojosa, J. A., and Mercado, F. (2001a) Emotion and attention interaction studied through event-related potentials. J. Cogn. Neurosci. 13, 1109-1128. doi: 10.1162/089892901753294400

Carretie, L., Mercado, F., Tapia, M., and Hinojosa, J. A. (2001b). Emotion, attention, and the negativity bias, studied through event-related potentials. Int. J. Psychophysiol. 41, 75-85. doi: 10.1016/s0167-8760(00)00195-1

Carroll, J. M., and Russell, J. A. (1996). Do facial expressions signal specific emotions? Judging emotion from the face in context. J. Pers. Soc. Psychol. 70, 205-218. doi: 10.1037/0022-3514.70.2.205

Cramer, P. (1998). Psychophysiology and expressed emotion. Br. J. Psychiatry 153:571.

Delorme, A., and Makeig, S. (2004). EEGLAB: an open source toolbox for analysis of single-trial EEG dynamics including independent component analysis. J. Neurosci. Methods 134, 9-21. doi: 10.1016/j.jneumeth.2003.10.009 
Eisenberg, N., Fabes, R. A., Guthrie, I. K., and Reiser, M. (2000). Dispositional emotionality and regulation: their role in predicting quality of social functioning. J. Pers. Soc. Psychol. 78, 136-157. doi: 10.1037/0022-3514.78.1.136

Etkin, A., Egner, T., and Kalisch, R. (2011). Emotional processing in anterior cingulate and medial prefrontal cortex. Trends Cogn. Sci. 15, 85-93. doi: 10.1016/j.tics.2010.11.004

Fazio, R. H., Sanbonmatsu, D. M., Powell, M. C., and Kardes, F. R. (1986). On the automatic activation of attitudes. J. Pers. Soc. Psychol. 50, 229-238. doi: 10.1037/0022-3514.50.2.229

Fox, E., Lester, V., Russo, R., Bowles, R. J., Pichler, A., and Dutton, K. (2000). Facial expressions of emotion: are angry faces detected more efficiently? Cognit. Emot. 14, 61-92. doi: 10.1080/026999300378996

Friedman, D., Cycowicz, Y. M., and Gaeta, H. (2001). The novelty P3: an event-related brain potential (ERP) sign of the brain's evaluation of novelty. Neurosci. Biobehav. Rev. 25, 355-373. doi: 10.1016/S0149-7634(01)00019-7

Gollwitzer, P. M., and Sheeran, P. (2006). Implementation intentions and goal achievement: a meta-analysis of effects and processes. Adv. Exp. Soc. Psychol. 38, 69-119. doi: 10.1016/S0065-2601(06)38002-1

Gross, J. J., and Levenson, R. W. (1993). Emotional suppression: physiology, self-report, and expressive behavior. J. Pers. Soc. Psychol. 64, 970-986. doi: 10.1037/0022-3514.64.6.970

Hallam, G. P., Webb, T. L., Sheeran, P., Miles, E., Wilkinson, I. D., Hunter, M. D., et al. (2015). The neural correlates of emotion regulation by implementation intentions. PLoS One 10:e0119500. doi: 10.1371/journal.pone.0119500

Herbert, C., Sfarlea, A., and Blumenthal, T. (2013). Your emotion or mine: labeling feelings alters emotional face perception-an ERP study on automatic and intentional affect labeling. Front. Hum. Neurosci. 7:378. doi: 10.3389/ fnhum.2013.00378

Herzmann, G., Bird, C. W., Freeman, M., and Curran, T. (2013). Effects of oxytocin on behavioral and ERP measures of recognition memory for ownrace and other-race faces in women and men. Psychoneuroendocrinology 38, 2140-2151. doi: 10.1016/j.psyneuen.2013.04.002

Hess, U., Blairy, S., and Kleck, R. E. (1997). The intensity of emotional facial expressions and decoding accuracy. J. Nonverbal Behav. 21, 241-257. doi: 10.1023/A:1024952730333

Hillyard, S. A., and Picton, T. W. (1987). "Electrophysiology of cognition" in handbook of physiology: Section 1, Neurophysiology. ed. F. Plum (New York, NY: Physiological Society), 519-584.

Jung, T. P., Makeig, S., Westerfield, M., Townsend, J., Courchesne, E., and Sejnowski, T. J. (2001). Analysis and visualization of single-trial event-related potentials. Hum. Brain Mapp. 14, 166-185. doi: 10.1002/hbm.1050

Klauer, K. C., and Musch, J. (2003). Affective priming: Findings and theories. Mahwah, NJ: Lawrence Erlbaum Associates Publishers.

Krombholz, A., Schaefer, F., and Boucsein, W. (2007). Modification of N170 by different emotional expression of schematic faces. Biol. Psychol. 76, 156-162. doi: 10.1016/j.biopsycho.2007.07.004

Lassalle, A., and Itier, R. J. (2013). Fearful, surprised, happy, and angry facial expressions modulate gaze-oriented attention: behavioral and ERP evidence. Soc. Neurosci. 8, 583-600. doi: 10.1080/17470919.2013.835750

Leppanen, J. M., and Hietanen, J. K. (2004). Positive facial expressions are recognized faster than negative facial expressions, but why? Psychol. Res. 69, 22-29. doi: 10.1007/s00426-003-0157-2

Levesque, J., Eugene, F., Joanette, Y., Paquette, V., Mensour, B., and Beaudoin, G. E. A. (2003). Neural circuitry underlying voluntary suppression of sadness. Biol. Psychiatry 53, 502-510. doi: 10.1016/S0006-3223(02)01817-6

Liang, X., Zebrowitz, L. A., and Zhang, Y. (2010). Neural activation in the reward circuit shows a nonlinear response to facial attractiveness. Soc. Neurosci. 5, 320-334. doi: 10.1080/17470911003619916

Luo, W. B., Feng, W. F., He, W. Q., Wang, N. Y., and Luo, Y. J. (2010). Three stages of facial expression processing: ERP study with rapid serial visual presentation. NeuroImage 49, 1857-1867. doi: 10.1016/j.neuroimage.2009.09.018

Lynn, S. K., and Salisbury, D. F. (2008). Attenuated modulation of the N170 ERP by facial expressions in schizophrenia. Clin. EEG Neurosci. 39, 108-111. doi: $10.1177 / 155005940803900218$

Mak, A. K., Hu, Z. G., Zhang, J. X., Xiao, Z. W., and Lee, T. M. (2009). Neural correlates of regulation of positive and negative emotions: an fmri study. Neurosci. Lett. 457, 101-106. doi: 10.1016/j.neulet.2009.03.094
Miyoshi, M., Katayama, J., and Morotomi, T. (2004). Face-specific N170 component is modulated by facial expressional change. Neuroreport 15, 911-914. doi: 10.1097/00001756-200404090-00035

Moors, A., and De Houwer, J. (2001). Automatic appraisal of motivational valence: motivational affective priming and Simon effects. Cognit. Emot. 15, 749-766. doi: 10.1080/02699930143000293

Nakajima, K., Minami, T., and Nakauchi, S. (2012). The face-selective N170 component is modulated by facial color. Neuropsychologia 50, 2499-2505. doi: 10.1016/j.neuropsychologia.2012.06.022

Ochsner, K. N., and Gross, J. J. (2005). The cognitive control of emotion. Trends Cogn. Sci. 9, 242-249. doi: 10.1016/j.tics.2005.03.010

Ochsner, K. N., Ray, R. D., Cooper, J. C., Robertson, E. R., Chopra, S., and Gabrieli, J. D. (2004). For better or for worse: neural systems supporting the cognitive down- and up-regulation of negative emotion. NeuroImage 23, 483-499. doi: 10.1016/j.neuroimage.2004.06.030

Ochsner, K. N., Silvers, J. A., and Buhle, J. T. (2012). Functional imaging studies of emotion regulation: a synthetic review and evolving model of the cognitive control of emotion. Ann. N. Y. Acad. Sci. 1251, E1-E24. doi: 10.1111/j.1749-6632.2012.06751.x

Olofsson, J. K., Nordin, S., Sequeira, H., and Polich, J. (2008). Affective picture processing: an integrative review of ERP findings. Biol. Psychol. 77, 247-265. doi: 10.1016/j.biopsycho.2007.11.006

Pascual-Marqui, R. D., Michel, C. M., and Lehmann, D. (1994). Low resolution electromagnetic tomography: a new method for localizing electrical activity in the brain. Int. J. Psychophysiol. 18, 49-65. doi: 10.1016/01678760(84)90014-X

Phan, K. L., Fitzgerald, D. A., Nathan, P. J., Moore, G. J., Uhde, T. W., and Tancer, M. E. (2005). Neural substrates for voluntary suppression of negative affect: a functional magnetic resonance imaging study. Biol. Psychiatry 57, 210-219. doi: 10.1016/j.biopsych.2004.10.030

Phillips, M. L., Ladouceur, C. D., and Drevets, W. C. (2008). A neural model of voluntary and automatic emotion regulation: implications for understanding the pathophysiology and neurodevelopment of bipolar disorder. Mol. Psychiatry 13, 829-857. doi: $10.1038 / \mathrm{mp} .2008 .65$

Pizzagalli, D. A., Oakes, T. R., Fox, A. S., Chung, M. K., Larson, C. L., and Abercrombie, H. C. (2004). Functional but not structural subgenual prefrontal cortex abnormalities in melancholia. Mol. Psychiatry 9, 393-405. doi: 10.1038/ sj.mp. 4001501

Recio, G., Shmuilovich, O., and Sommer, W. (2014). Should I smile or should I frown? An ERP study on the voluntary control of emotion-related facial expressions. Psychophysiology 51, 789-799. doi: 10.1111/psyp.12220

Righart, R., and de Gelder, B. (2006). Context influences early perceptual analysis of faces-an electrophysiological study. Cereb. Cortex 16, 1249-1257. doi: 10.1093/cercor/bhj066

Roca, P., Mulas, F., Ortiz-Sanchez, P., and Gandia-Beneto, R. (2015). Emotional self-regulation in infantile attention deficit hyperactivity disorder and P300 evoked potentials. Rev. Neurol. 60, S69-S74.

Ruz, M., Madrid, E., and Tudela, P. (2013). Interactions between perceived emotions and executive attention in an interpersonal game. Soc. Cogn. Affect. Neurosci. 8, 838-844. doi: 10.1093/scan/nss080

Trautmann, S. A., Fehr, T., and Herrmann, M. (2009). Emotions in motion: dynamic compared to static facial expressions of disgust and happiness reveal more widespread emotion-specific activations. Brain Res. 1284, 100-115. doi: 10.1016/j.brainres.2009.05.075

Vlamings, P. H., Goffaux, V., and Kemner, C. (2009). Is the early modulation of brain activity by fearful facial expressions primarily mediated by coarse low spatial frequency information? J. Vis. 9, 12-13. doi: $10.1167 / 9.5 .12$

Werheid, K., Alpay, G., Jentzsch, I., and Sommer, W. (2005). Priming emotional facial expressions as evidenced by event-related brain potentials. Int. J. Psychophysiol. 55, 209-219. doi: 10.1016/j.ijpsycho.2004.07.006

Williams, L. L., Bahramali, H., Hemsley, D. R., Harris, A. W., Brown, K., and Gordon, E. (2003). Electrodermal responsivity distinguishes ERP activity and symptom profile in schizophrenia. Schizophr. Res. 59, 115-125. doi: 10.1016/S0920-9964(01)00368-1

Williams, L. M., Palmer, D., Liddell, B. J., Song, L., and Gordon, E. (2006). The when and where of perceiving signals of threat versus non-threat. NeuroImage 31, 458-467. doi: 10.1016/j.neuroimage.2005.12.009 
Wu, Y., and Zhou, X. (2009). The P300 and reward valence, magnitude, and expectancy in outcome evaluation. Brain Res. 1286, 114-122. doi: 10.1016/j. brainres.2009.06.032

Yang, Q., Zhao, D., Wu, Y., Tang, P., Gu, R., and Luo, Y. J. (2018). Differentiating the influence of incidental anger and fear on risk decision-making. Physiol. Behav. 184, 179-188. doi: 10.1016/j.physbeh.2017.11.028

Yuan, L., Zhou, R., and Hu, S. (2014). Cognitive reappraisal of facial expressions: electrophysiological evidence of social anxiety. Neurosci. Lett. 577, 45-50. doi: 10.1016/j.neulet.2014.06.006

Zhang, D., Luo, W., and Luo, Y. (2013). Single-trial ERP analysis reveals facial expression category in a three-stage scheme. Brain Res. 1512, 78-88. doi: 10.1016/j.brainres.2013.03.044

Zumsteg, D., Friedman, A., Wieser, H. G., and Wennberg, R. A. (2006). Propagation of interictal discharges in temporal lobe epilepsy: correlation of spatiotemporal mapping with intracranial foramen ovale electrode recordings. Clin. Neurophysiol. 117, 2615-2626. doi: 10.1016/j.clinph.2006.07.319

Conflict of Interest: The authors declare that the research was conducted in the absence of any commercial or financial relationships that could be construed as a potential conflict of interest.

Copyright (c) 2020 Yang, Zhang, Wang and Wu. This is an open-access article distributed under the terms of the Creative Commons Attribution License (CC $B Y)$. The use, distribution or reproduction in other forums is permitted, provided the original author(s) and the copyright owner(s) are credited and that the original publication in this journal is cited, in accordance with accepted academic practice. No use, distribution or reproduction is permitted which does not comply with these terms. 